\title{
The effect of Gabapentin on the expression of genes in the trigeminal ganglia of inferior alveolar nerve-transected neuropathic pain model rats
}

\author{
Masayo Okumura ${ }^{1}$ and Eiji Kondo ${ }^{1,2}$ \\ ${ }^{1}$ Institute for Oral Science and ${ }^{2}$ First Department of Oral Anatomy, \\ Matsumoto Dental University
}

[Received 8 February 2010, Accepted 5 July 2010]

\begin{abstract}
Gabapentin, an antiepileptic drug, selectively interacts with alpha-2 / delta 1 calcium channel subunits (CACNA2D1), and has an analgesic effect on neuropathic pain. Neuropathic pain is known to be accompanied by the up-regulation of the expression of many genes. We observed the involvement of the analgesic effect of gabapentin and the alteration of the expression of genes in an inferior alveolar nerve-transected rat neuropathic pain model, in which IAN transection induced allodynia in whisker pads innervated by uninjured neurons.

We determined the up-regulation of the expression of several genes in the trigeminal ganglia of IAN-transected rats, including those for neuropeptides, GDNF receptor and CACNA2D1; however, these up-regulations were confined almost exclusively to injured neurons, and were not detected in the neurons innervating the allodynia area. Upon suppression of tactile allodynia in IAN-transected rats by intrathecal administration of gabapentin, the increased gene expression levels did not change, other than that of CACNA2D1, whose expression was reduced with the attenuation of allodynia.

These findings indicate that gabapentin relieves the allodynia developed in the cutaneous region innervated by uninjured neurons, but that the main target of gabapentin is the CACNA2D1 expressed in injured neurons.
\end{abstract}

Key words: Neuropathic pain; Gabapentin; Gene expression; CACNA2D1

PAIN RESEARCH 25 (2010) 171-178

\section{INTRODUCTION}

Nerve injury-induced neuropathic pain is a type of chronic pain characterized by mechanical and thermal hyperalgesia and allodynia, which develop in a wide area and persist for a long time ${ }^{1)}$. Inferior alveolar nerve-transected rats are one type of neuropathic pain model rats, in which the modeled disorder is induced by the transection of 
the inferior alveolar nerve (IAN). Although the transected IAN is a component of the mandibular nerve in the trigeminal ganglion (TG), hypersensitivity to mechanical stimulation developed in the whisker pads, which are innervated by the uninjured infraorbital nerve, a component of the maxillary nerve in the $\mathrm{TG}^{21)}$.

Nerve injury is known to be associated with the up-regulation of the expression of many genes in the ganglion neurons, including those for neuropeptides ${ }^{16)}$, interleukins ${ }^{15)}$ and transcription factors ${ }^{22}$. CACNA2D1, encoding the alpha-2 / delta 1 $(\alpha 2 \delta-1)$ subunit of voltage-dependent calcium channels, is also up-regulated in neurons by nerve injury ${ }^{13)}$, and is thought to play an important role in the pathogenesis of hypersensitivity in neuropathic pain because it is a target molecule of gabapentin ${ }^{20)}$.

Gabapentin, which binds to CACNA2D1 and inhibits the calcium influx through the channel, is a first-line drug for neuropathic pain ${ }^{4)}$. Although tactile allodynia can be developed in many neuropathic pain models, the effectiveness of gabapentin for tactile allodynia was observed only in models with significant $\alpha 2 \delta-1$ subunit up-regulation in ganglion neurons ${ }^{12)}$. These models confirm the important role of the calcium influx in the pathogenesis of injury-dependent tactile allodynia.

In this study, we analyzed the effect of gabapentin in IAN-injury model rats. Tactile allodynia in the whisker pads and the up-regulation of the gene expression of CACNA2D1, among other molecules, in TG neurons induced by IAN injury were observed. The results reveal that gabapentin is efficacious in treating tactile allodynia in IAN-injury model rats, and that the gene expression of CACNA2D1 is specifically co-regulated with tactile allodynia.

\section{MATERIALS AND METHODS}

\section{Animals and surgery}

Sixty-one 6 8-week-old male Sprague Dawley rats were used in this study. All surgeries and treatments were carried out in accordance with the ethical guidelines of Matsumoto Dental University.

For the nerve injury surgery, the inferior alveolar nerve (IAN) on the unilateral side of the rats was axotomized. Briefly, the rats were anesthetized with sodium pentobarbital (Dainippon Sumitomo Pharma), and the surface of the alveolar bone was exposed through a small incision of the facial skin and masseteric muscle. The bone surface covering the IAN was removed, a small piece (1 mm in length) of the exposed IAN was transected and removed, and the masseteric muscle and skin were sutured.

Two weeks before the IAN surgery, the spinal subarachnoid space was cannulated for the intrathecal administration of gabapentin (Sigma). After the anesthetized rats were placed in a stereotaxic frame (Narishige), the atlanto-occipital membrane was exposed by removing the overlying muscle through a dorsal mid-line incision. A 65-mm long $\mathrm{PE}-10$ tube filled with saline was introduced, $10 \mathrm{~mm}$ of it under the dura mater through a small cut in the atlantooccipital membrane, and $55 \mathrm{~mm}$ of it left at the dorsal surface after suturing the muscle and skin. Gabapentin $(20 \mu \mathrm{l} ; 10$ $\mathrm{mg} / \mathrm{ml}$ in saline) or saline $(20 \mu \mathrm{l})$ was injected through the cannulated tube once a day after the daily behavioral test.

\section{Behavioral test}

After habituation to the environment and instrumentation, tactile allodynia was assessed by measuring the withdrawal 
thresholds with a series of graded von Frey filaments. A series of calibrated von Frey filaments were applied to the skin of the whisker pad of each rat, which was immobilized and blinded by hand. A medium-sized filament was applied perpendicularly until the filament bent slightly. If the rat withdrew its face upon the application of the filament, then a filament one size smaller was tested. Conversely, if no response was observed, a filament one size larger was tested. In this way, the minimum filament size (in grams) required to induce active withdrawal of the head from the probing filament was defined as the threshold inducing a response. When the thresholds during 3 days after the IAN operation were significantly decreased compared to those of 3 days before the operation, the rat was identified as developing mechanical allodynia. In this study, a little under $50 \%$ of the IAN transected rats developed the mechanical allodynia, and such the rats were used for the analysis.

\section{Real-time PCR}

The trigeminal ganglia were removed and separated by using tweezers to reach the mandibular (V3) region and the ophthalmic and maxillary (V1V2) region. Each piece of tissue was immediately frozen in liquid nitrogen, and total RNA samples were extracted using TRIZOL (Invitrogen), in accordance with the manufacturer's protocol. The synthesized cDNAs were diluted to ten times the original volume using sterile water, and $0.5 \mu \mathrm{l}$ of the diluted cDNA was used for each reaction. The primers and TaqMan probe (5'-FAM, and 3'-TAMRA conjugate) for each gene were designed by the Primer3 program ${ }^{18)}$, and synthesized (Nihon Bioservice). Real-time PCR was performed using Premix Ex Taq ${ }^{\mathrm{TM}}$ (Perfect Real Time) (TaKaRa) in accordance with

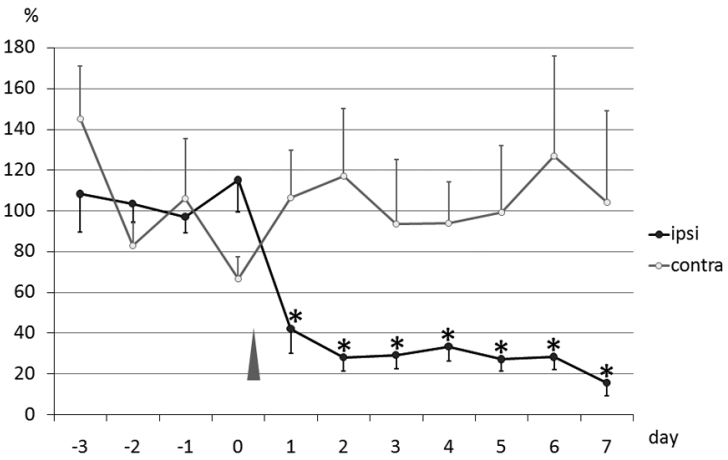

Fig.1 Tactile allodynia induced by IAN transection. The tactile withdrawal thresholds in the rats' whisker pads on the ipsilateral and contralateral sides were compared to the average threshold over a period of 3 days before transection, which is defined as $100 \%$. The arrowhead indicates the day of operation. n=9. Asterisks : $p<0.05$, Wilcoxon-MannWhitney test.

the manufacturer's protocol, and the data were collected and analyzed by a DNA Engine Opticon ${ }^{\mathrm{TM}}$ System (Bio-Rad).

\section{RESULTS}

\section{Tactile allodynia and gene expression after IAN transection}

The withdrawal thresholds for tactile stimulation in the ipsilateral-side whisker pads were significantly reduced from day 1 after IAN transection compared to the baseline (Fig.1). To analyze the alterations of gene expression in the trigeminal neurons accompanying developing allodynia, the trigeminal ganglia after IAN transection over a period of 7 days were harvested and separated into two pieces, the V3 region and the V1V2 region. The V3 region of the trigeminal ganglion contains the cell bodies of the mandibular nerve, and the transected IAN is a component of the mandibular nerve. On the other hand, the V1V2 region contains the cell bodies of the infraorbital nerve, which innervates the allodynia-affected 

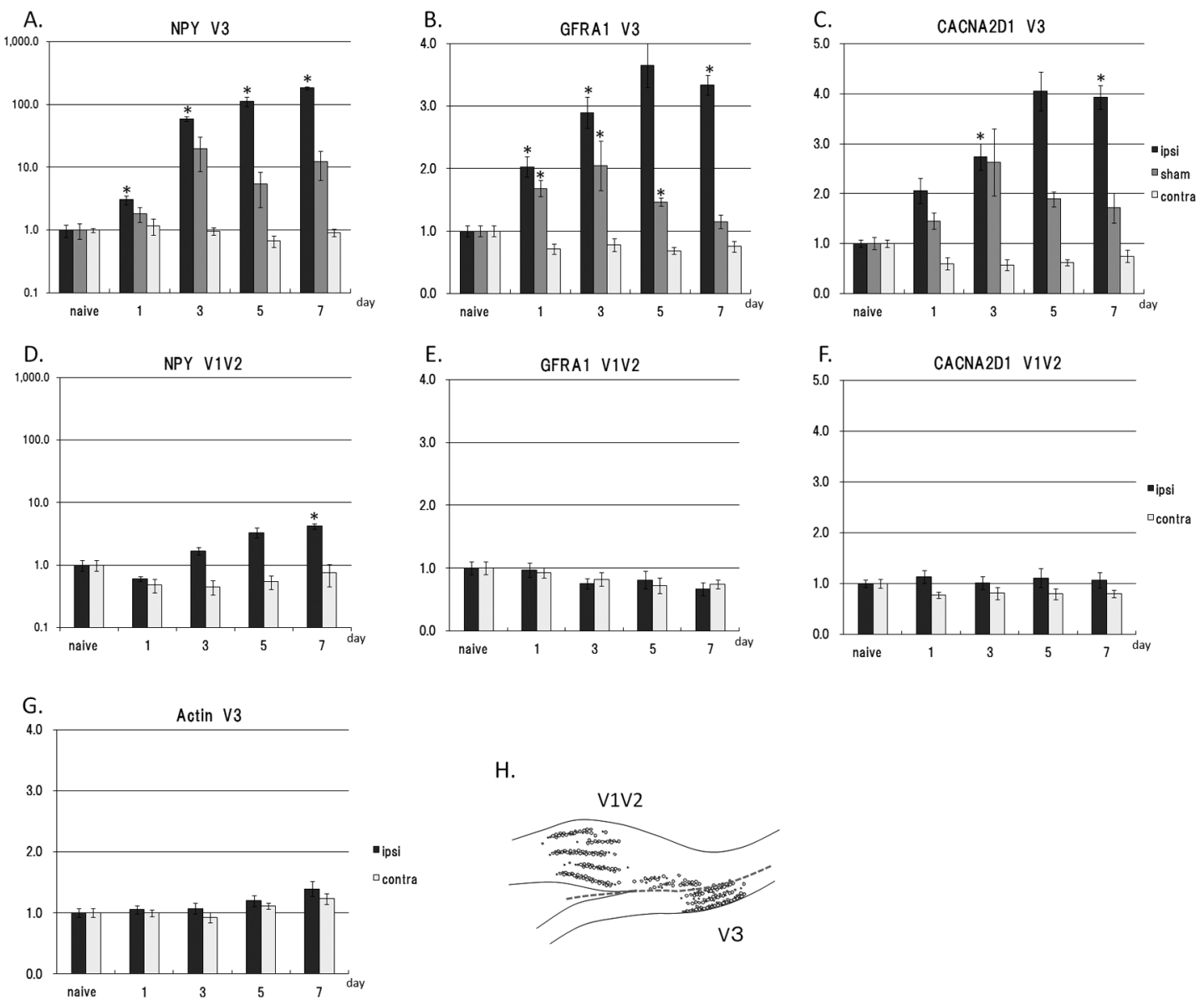

H.

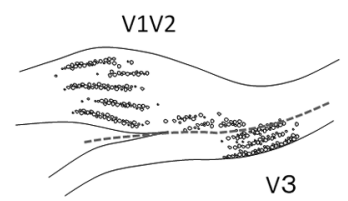

Fig.2 Alterations of gene expression after IAN transection.

The amounts of the indicated mRNAs in the V3 region and the V1V2 region of the TG over a period of 7 days after IAN transection were analyzed by real-time PCR, and compared to the data for naive rats (A - G). The mRNAs of GAL and VIP showed alteration patterns similar to those of NPY (data not shown). The TG was separated into V3 and V1V2 regions as shown (H). Significant differences to naive rats are indicated by asterisks (Wilcoxon-Mann-Whitney test, $\mathrm{p}<0.01$ ). $\mathrm{n}=4$ for naive, and $\mathrm{n}=6$ for day 1 , day 3 , day 5 , and day 7 .

whisker pads ${ }^{17)}$. Real-time PCR analysis revealed the up-regulation of neuropeptide $\mathrm{Y}$ (NPY), galanin (GAL), vasoactive intestinal peptide (VIP), glial cell line-derived neurotrophic factor receptor alpha 1 (GFRA1) and CACNA2D1 in the V3 region of the ipsilateral-side trigeminal ganglion from 1 day after IAN transection, but not in the contralateral-side V3 region or V1V2 region (Fig.2). These genes also showed up-regulation upon sham operation, but this change was tran- sient. The actin gene did not show any change in expression. Although NPY showed a slight up-regulation in the V1V2 region, it can be interpreted as indicating the incomplete separation of the TG into the V3 and V1V2 regions. The analysis of the V3 region showed vast NPY expression in the injured inferior alveolar neurons, and majority of the injured neurons localized to in V3 region in TG, but a small minority will spill into the V1V2 region. 


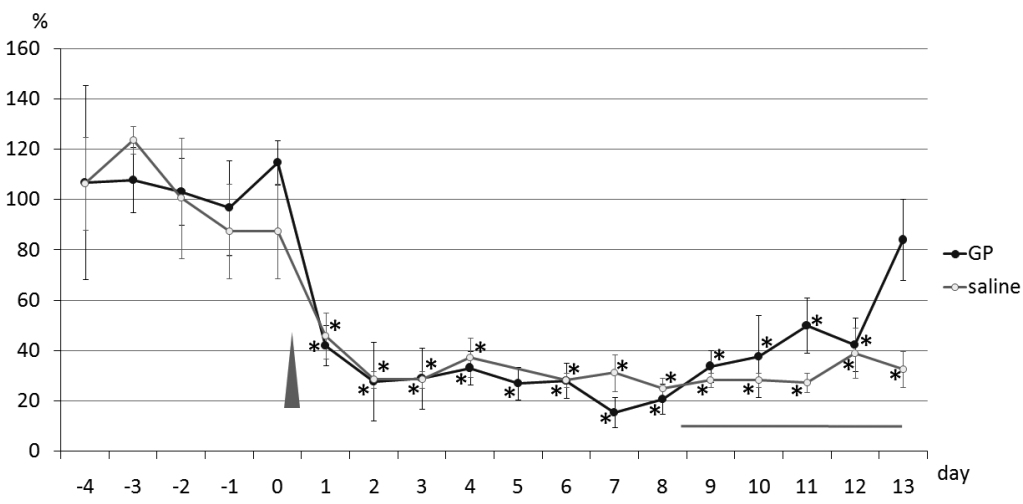

Fig.3 Reduction of tactile allodynia by gabapentin.

The tactile withdrawal thresholds of the rats' ipsilateral side whisker pads were compared to the average threshold over a period of 3 days before transection, which is defined as 100\%. Allodynia was induced by IAN transection (arrowhead), and reduced by gabapentin administration, not by saline (bar). Asterisk: compared to the average threshold over a period of 3 days before transaction ( $\mathrm{p}<0.05$, Wilcoxon-Mann-Whitney test). $\mathrm{n}=9$ for gabapentin, and $\mathrm{n}=4$ for saline.
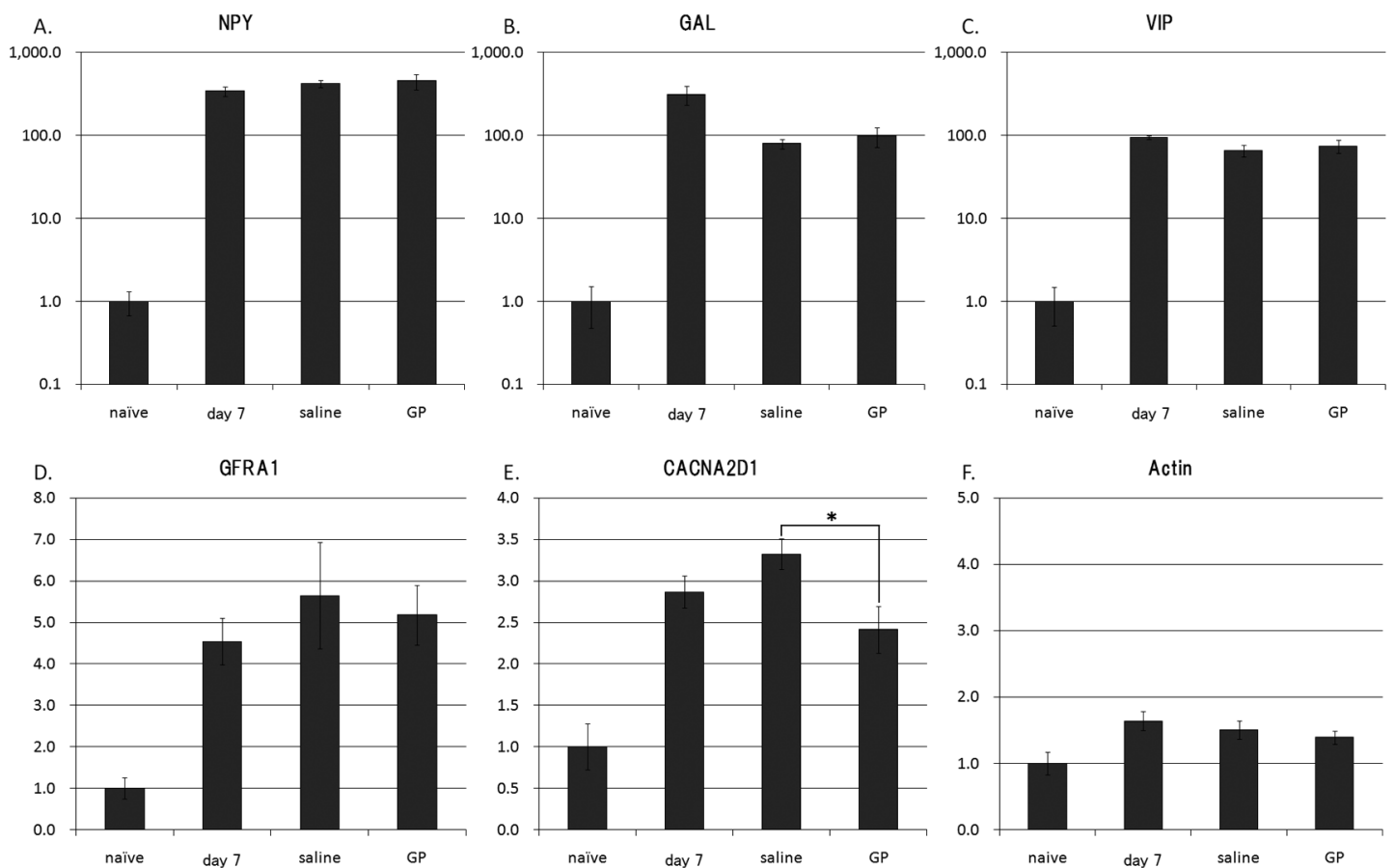

Fig.4 Effect of gabapentin on gene expression.

The expression of genes in the V3 region of the TG was analyzed 7 days after IAN transection (day 7), after saline administration (saline) or gabapentin administration (GP), and compared to the naive data (naive). $\mathrm{n}=9$, asterisk: compared to saline, $\mathrm{p}<0.05$ (Wilcoxon-Mann-Whitney test).

\section{Effect of gabapentin}

Gabapentin was intrathecally injected into the dorsal medulla of the rats under allodynia induced by IAN transection. The tactile thresholds reduced by IAN transection were gradually restored by daily gabapentin injection (Fig.3), despite the fact that the allodynia of this model rat has been reported to persist for over a 
month ${ }^{9)}$. The expression of genes up-regulated by IAN transection in the V3 region of the TG was analyzed again after 5 administrations of gabapentin or saline. Compared to the saline injection, gabapentin significantly inhibited the CACNA2D1 gene expression, but did not inhibit the expression of the other genes (Fig.4).

\section{DISCUSSION}

Among the many rat models of neuropathic pain, the IAN transection model has the advantage of capturing the morphological features of the TG, in which the cell bodies of the V3 nerve and the V1V2 nerve are disproportionately localized ${ }^{17)}$; thus, the injured neurons and the neurons innervating the allodynia area can be analyzed separately. In this study, we analyzed the gene expression of NPY, VIP, GAL 16), GFRA1 ${ }^{11)}$ and CACNA2D1 ${ }^{13)}$ in the $\mathrm{V} 3$ and $\mathrm{V} 1 \mathrm{~V} 2$ regions of the TG after IAN injury. All of these genes have been reported to be up-regulated in the dorsal root ganglion neurons after sciatic nerve injury. Our results indicate that their expression was indeed up-regulated in the injured neurons of the V3 region, but not in the neurons of the V1V2 region innervating the allodynia area. This finding was unexpected, considering the previous report on the deep involvement of the expression of these genes in allodynia pathogeny.

Gabapentin, an antiepileptic drug, binds to CACNA2D1 and inhibits the calcium influx through the channel ${ }^{6,19)}$. The antineuropathic effect of gabapentin also arises from its channel-inhibiting property ${ }^{5)}$. The fact that gabapentin has an anti-neuropathic effect only in models with signifi- cant CACNA2D1 mRNA up-regulation in the ganglion neurons ${ }^{12)}$ indicates the important role of the hyperexcitability of neurons associated with calcium influx and signaling. It also indicates that the major target of gabapentin is the $\alpha 2 \delta-1$ subunit translated from mRNA additively transcribed after nerve injury. In our results, the efficacy of gabapentin in the IAN transection model suggests that the underlying mechanisms of whisker pad allodynia are the same as or similar to those of the previously reported hind paw allodynia in the sciatic nerve model. In addition, it is also suggested that the hyperexcitable neurons, which are responsible for tactile allodynia and targeted by gabapentin, are the injured neurons in the V3 region, not the neurons in the V1V2 region innervating the allodynia area, because the up-regulation of CACNA2D1 was detected only in the V3 region of the TG, not in the V1V2 region. Because gabapentin inhibits the calcium influx through the channel containing CACNA2D1 subunit ${ }^{6,19)}$, the transcription of the CACNA2D1 gene in the injured neurons can be speculated to be regulated under the function of CACNA2D1 itself, probably through calcium signaling spreading from the synapses in the medullary dorsal horn, where the CACNA2D1 protein localize ${ }^{14)}$.

All the genes analyzed here have been previously reported to be up-regulated in neuropathic pain model rats ${ }^{11,13,16)}$, and to be associated with the pathogeny of neuropathic pain ${ }^{2,3,7,8,10)}$, based on synchronized changes in their expression levels and the tactile threshold of the rats. However, we detected only CACNA2D1 down-regulation following the recovery of the tactile threshold induced by gabapentin. Other genes, such as NPY, GAL, VIP and GFRA1, were up-regulated after IAN injury, but not 
down-regulated by gabapentin administration. These results suggest that the upregulation of these genes is not necessarily essential for the persistence of neuropathic pain. However, the precise roles of these genes in the generation, persistence and recovery of allodynia are still unclear, because we observed only the effect of gabapentin administration after the generation of allodynia. We have poor knowledge about the mechanisms behind the development and persistence of allodynia, and the functional mechanism of gabapentin. The elucidation of the significance of the up- and down-regulation of these genes in neurons, especially CACNA2D1, will require further analysis.

\section{Acknowledgments}

The authors thank Toshizo Ishikawa, professor at the School of Allied Health Sciences, Yamaguchi University, for his instructions on the cannulation technique. This work was supported by a Grant-in-Aid for Young Scientists (B) (19791554) from the Ministry of Education, Culture, Sports, Science and Technology of Japan.

\section{References}

1) Baron, R., Mechanisms of Disease: neuropathic pain - a clinical perspective, Nature Clinical. Practice Neurology, 2 (2006) 95-106.

2) Boroujerdi, A., Kim, H.K., Lyu, Y.S., Kim, D.S., Figueroa, K.W., Chung, J.M., Luo, Z.D., Injury discharges regulate calcium channel alpha-2-delta1 subunit upregulation in the dorsal horn that contributes to initiation of neuropathic pain, Pain, 139 (2008) 358-366.

3) Dong, Z.Q., Ma, F., Xie, H., Wang, Y.Q., Wu, G.C., Down-regulation of GFRalpha-1 expression by antisense oligodeoxynucleotide attenuates electroacupuncture analgesia on heat hyperalgesia in a rat model of neuropathic pain, Brain Res. Bull., 69 (2006) 30-36.
4) Dworkin, R.H., O'Connor, A.B., Backonja, M., Farrar, J.T., Finnerup, N.B., Jensen, T.S., Kalso, E.A., Loeser, J.D., Miaskowski, C., Nurmikko, T.J., Portenoy, R.K., Rice, A.S., Stacey, B.R., Treede, R.D., Turk, D.C., Wallace, M.S., Pharmacologic management of neuropathic pain: evidence-based recommendations, Pain, 132 (2007) 237-251.

5) Field, M.J., Cox, P.J., Stott, E., Melrose, H., Offord, J., Su, T.-Z., Bramwell, S., Corradini, L., England, S., Winks, J., Kinloch, R.A., Hendrich, J., Dolphin, A.C., Webb, T., Williams, D., Identification of the alpha-2-delta-1 subunit of voltage-dependent calcium channels as a molecular target for pain mediating the analgesic actions of pregabalin, Proc. Nat. Acad. Sci., 103 (2006) 17537-17542.

6) Gee, N.S., Brown, J.P., Dissanayake, V.U., Offord, J., Thurlow, R., Woodruff, G.N., The novel anticonvulsant drug, gabapentin (Neurontin), binds to the alpha2delta subunit of a calcium channel, J. Biol. Chem., 271 (1996) 5768-5776.

7) Holmes, F.E., Mahoney, S.A., Wynick, D., Use of genetically engineered transgenic mice to investigate the role of galanin in the peripheral nervous system after injury, Neuropeptides, 39 (2005) 191199.

8) Intondi, A.B., Dahlgren, M.N., Eilers, M.A., Taylor, B.K., Intrathecal neuropeptide Y reduces behavioral and molecular markers of inflammatory or neuropathic pain, Pain, 137 (2008) 352-365.

9) Iwata, K., Imai, T., Tsuboi, Y., Tashiro, A., Ogawa, A., Morimoto, T., Masuda, Y., Tachibana, Y., Hu, J., Alteration of medullary dorsal horn neuronal activity following inferior alveolar nerve transection in rats, J. Neurophysiol., 86 (2001) 2868-2877.

10) Jeftinija, S., Murase, K., Nedeljkov, V., Randic, M., Vasoactive intestinal polypeptide excites mammalian dorsal horn neurons both in vivo and in vitro, Brain Res., 243 (1982) 158-164.

11) Kashiba, H., Hyon, B., Senba, E., Glial cell linederived neurotrophic factor and nerve growth factor receptor mRNAs are expressed in distinct subgroups of dorsal root ganglion neurons and are differentially regulated by peripheral axotomy in the rat, Neurosci. Lett., 252 (1998) 107-110.

12) Luo, Z.D., Calcutt, N.A., Higuera, E.S., Valder, C.R., Song, Y.H., Svensson, C.I., Myers, R.R., Injury type-specific calcium channel alpha 2 delta1 subunit up-regulation in rat neuropathic pain models correlates with antiallodynic effects of gabapentin, J. Pharmacol. Exp. Ther., 303 (2002) 1199-1205. 
13) Luo, Z.D., Chaplan, S.R., Higuera, E.S., Sorkin, L.S., Stauderman, K.A., Williams, M.E., Yaksh, T.L., Upregulation of dorsal root ganglion (alpha)2(delta) calcium channel subunit and its correlation with allodynia in spinal nerve-injured rats, J. Neurosci., 21 (2001) 1868-1875.

14) Melrose, H.L., Kinloch, R.A., Cox, P.J., Field, M.J., Collins, D., Williams, D., $[3 \mathrm{H}]$ pregabalin binding is increased in ipsilateral dorsal horn following chronic constriction injury, Neurosci. Lett., 417 (2007) 187-192.

15) Murphy, P.G., Grondin, J., Altares, M., Richardson, P.M., Induction of interleukin-6 in axotomized sensory neurons, J. Neurosci., 15 (1995) 5130-5138.

16) Noguchi, K., De León, M., Nahin, R.L., Senba, E., Ruda, M.A., Quantification of axotomy-induced alteration of neuropeptide mRNAs in dorsal root ganglion neurons with special reference to neuropeptide Y mRNA and the effects of neonatal capsaicin treatment, J. Neurosci. Res., 35 (1993) 54-66.

17) Phil M.E. Waite, David J. Tracey, 27-Trigeminal Sensory System, In : George Paxins (Ed), The Rat Nervous System second edition, Academic Press, California, 1995, pp705-724.

18) Rozen, S., Skaletsky, H.J., Primer3 on the WWW for general users and for biologist programmers, In: Krawetz S, Misener S (Eds), Bioinformatics Methods and Protocols: Methods in Molecular Biology, Humana Press, Totowa, NJ, 2000, pp365386.
19) Sutton, K.G., Martin, D.J., Pinnock, R.D., Lee, K., Scott, R.H., Gabapentin inhibits high-threshold calcium channel currents in cultured rat dorsal root ganglion neurons, Br. J. Pharmacol., 135 (2002) 257-265.

20) Taylor, C.P., Mechanisms of analgesia by gabapentin and pregabalin - calcium channel alpha2-delta [Cavalpha2-delta] ligands, Pain, (2009) 13-16.

21) Tsuboi, Y., Takeda, M., Tanimoto, T., Ikeda, M., Matsumoto, S., Kitagawa, J., Teramoto, K., Simizu, K., Yamazaki, Y., Shima, A., Ren, K., Iwata, K., Alteration of the second branch of the trigeminal nerve activity following inferior alveolar nerve transection in rats, Pain, 111 (2004) 323334.

22) Tsujino, H., Kondo, E., Fukuoka, T., Dai, Y., Tokunaga, A., Miki, K., Yonenobu, K., Ochi, T., Noguchi, K., Activating transcription factor 3 (ATF3) induction by axotomy in sensory and motoneurons: A novel neuronal marker of nerve injury, Mol. Cell Neurosci., 15 (2000) 170-182.

Address for correspondence: Masayo Okumura

Institute for Oral Science, Matsumoto Dental University

1780 Gobara Hirooka, Shiojiri, Nagano 399-0781, Japan

Tel: 0263-51-2183 / Fax: 0263-53-3456 / E-mail: okmrm@ po.mdu.ac.jp 